Proceedings of the 35th European Peptide Symposium

\title{
Combinatorially screened peptide as targeted covalent binder
}

\author{
Shuta Uematsu' ${ }^{1}$, Yudai Tabuchi ${ }^{1}$, Yuji Ito ${ }^{2}$, Masumi Taki ${ }^{1}$ \\ ${ }^{1}$ The University of Electro-Communications, Japan \\ ${ }^{2}$ Graduate School of Science and Engineering, Kagoshima University, Japan
}

https://doi.org/10.17952/35EPS.2018.272

Finding targeted covalent binders (also known as covalent drugs) is one of the cutting-edge disciplines such as biomedical sciences / chemical biology / pharmaceuticals fields. As shown in the figure below, we present here a novel concept for finding a peptide covalent binder from fluoroprobe-modified peptide library [1] on T7 bacteriophage constructed via the 10BASE $\mathrm{d}_{\mathrm{d}} \mathrm{T}$ [2], followed by a structure alteration into a reactive warhead [3]. Site- and position-specific conjugation toward the target protein and target selectivity were confirmed. This is the first demonstration for finding peptide-type targeted covalent binder by a combinatorial screening, instead of a rational designing. We believe the discovery technology would be generally applied to the discovery of novel covalent drugs for clinically-important proteins (e.g., cancer-related cell-surface proteins).
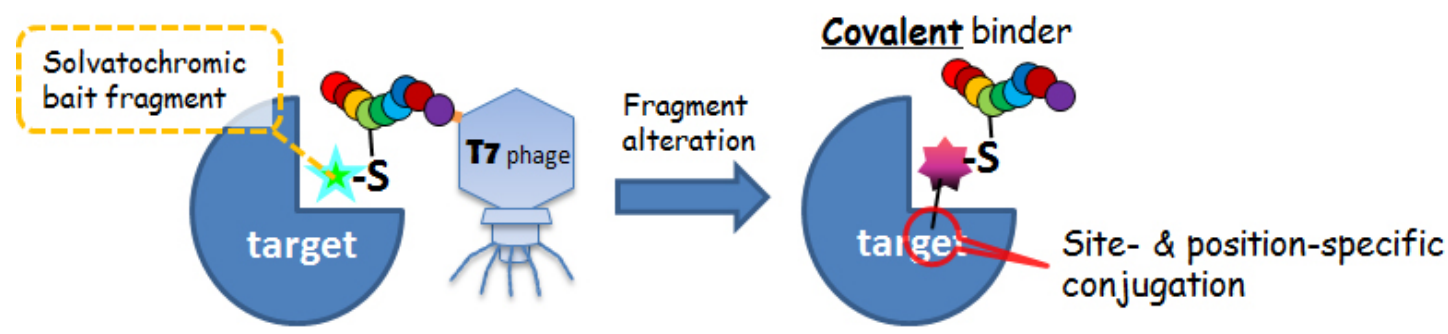

Figure 1

\section{References}

[1] Taki et al., Anal. Chem. 2016, 88, 1096; Uematsu et al., AIP Conf. Proc. 2017, 1807, 020028.

[2] Fukunaga et al., Chem. Commun. 2014, 50, 3921; inside cover article.

[3] Uematsu and Tabuchi et al., Bioconj. Chem. 2018, in press. 